\title{
Theory of free-carrier absorption in the presence of a quantizing magnetic field in quasi-one-dimensional quantum well structures
}

\author{
G.B. Ibragimov \\ Institute of Physics, NAS of Azerbaijan Republic, 33, Javid av., 1143 Baku, Azerbaijan \\ E-mail:guseyn@physics.ab.az and guseyn_gb@mail.ru
}

\begin{abstract}
The theory of free-carrier absorption is given for a quasi one-dimensional semiconducting structures in a quantizing magnetic field for the case when carriers are scattered by polar optical phonons and acoustic phonons and the radiation field is polarized perpendicular to the magnetic field direction. The usual resonance condition $P \omega_{c}=\Omega+\omega_{0}$, where $P$ is an integer and $w_{0}$ and $w_{c}$ are the optical-phonon frequency and cyclotron frequency, respectively, becomes $P \omega=\Omega+\omega_{0}$ with $\widetilde{\omega}$ equal to $\sqrt{\omega_{c}^{2}+\omega^{2}}$. The magnetic field dependence of the absorption for the transverse configuration can be explained in terms of phonon-assisted transitions between various Landau levels of carriers.
\end{abstract}

Keywords: quantizing magnetic field, quantum well.

Paper received 18.03.04; accepted for publication 21.10.04.

\section{Introduction}

The application of magnetic field to crystal changes the dimensionality of electronic levels and leads to a redistribution of the density of states. Quantum well wires $(\mathrm{QWW})$ in a magnetic field have been the subject of a few investigations [1-5]. In [1] rectangular QWWs were treated in the decoupled approximation. Concerning the theoretical work on magnetotransport in QWWs, we are aware of the Hall resistivity treatments of Refs 2 and 3 and of magnetophonon oscillations in Ref. 4. In [5] the fieldinduced change in optical anisotropy was studied for a quasi - two- dimensional (Q2D) system subject to a periodic modulation. The effect of a magnetic field on conductance quantization in quasi-one-dimensional (Q1D) systems is reviewed in [6]. An essential progress in techniques of growth on patterned substrates and of cleavededge overgrowth has led to QWWs with very good optical properties [7-10], thus renewing the interest for the basic properties of Q1D systems.

In this work, we are interested in the effect of magnetic field on the free carrier absorption (FCA) in semiconductor QWWs. Over the past two decades the investigation of FCA in low-dimensional systems has been very intense. FCA is one of the powerful means to understand the scattering mechanisms of carriers. In bulk semiconductors, it accounts for the absorption of electromagnetic radiation of frequencies $\Omega$ such that $\hbar \Omega<E_{g}$, where $E_{g}$ is the band gap [11]. In quantum well (QW) structures, apart from the direct interband and intersubband optical transitions, the optical absorption can also take plase via indirect intrassubband optical transitions in which carriers absorb or emit photons with a simultaneous scattering by phonons or other imperfections. The quantum theory for FCA in Q2D structures is well developed both in the absence [12-22] and in the presence of quantizing magnetic fields [23]. In the works [23], we have extended the theory of FCA in Q2D systems in the presence of a quantizing magnetic field when phonon scattering is important, and it was found that FCA coefficient oscillates as a function of the magnetic field and photon frequency with resonances occuring when $P \omega_{c}=\Omega \pm \omega_{0}$, where $\omega_{c}, \Omega$ and $\omega_{0}$ are the cyclotron, photon and phonon frequencies, respectively, and where $P$ is an integer. The theory FCA has been studied theoretically in quasi-one dimensional (Q1D) structures only in the absence of quantizing magnetic field [24-27].

In this paper, we extend the quantum theory of FCA developed previously to take into account the presence of quantizing magnetic fields. We consider FCA for the case 


\section{G.B. Ibragimov: Theory of free-carrier absorption in the presence of ...}

when carriers are scattered by the alloy disorder, acoustic phonons and boundary roughness. We will present a calculation of FCA coefficient for electromagnetic radiation polarized along the length of the wire. The magnetic field is assumed to be perpendicular to the wire axis, so that the dispersion of one-dimensional subbands is strongly modified.

\section{Formalism}

We consider Q1D electron gas confined in a wire of dimensions $L_{x}, L_{y}, L_{z}$. We model transverse confinement via an infinite square well approximation to a heterojunction quantum well ( $z$ axis) and a parabolic potential of frequency $\omega$ ( $x$ axis). Moreover, a magnetic field $\mathrm{B}$, parallel to the $z$ axis, is applied to the wire. The electrons are free in the direction of the wire ( $y$ axis). Correspondingly, the one-electron eigenfunctions $\Psi_{N l k_{y}}$ and energy eigenvalues $E_{N l k_{y}}$ are given by

$$
\begin{aligned}
& \Psi_{N l k_{y}}=\left(\frac{2}{L_{y} L_{z}}\right)^{1 / 2} \Phi_{N}\left(x-x_{0}\right) e^{i k_{y} y} \sin \left(\frac{l \pi z}{L_{z}}\right) \\
& E_{N l k_{y}}=\left(N+\frac{1}{2}\right) \hbar \widetilde{\omega}_{c}+\frac{\hbar^{2} k_{y}^{2}}{2 \widetilde{m}^{*}}+l^{2} E_{0}
\end{aligned}
$$

where $N=0,1,2, \ldots, l=1,2,3, \ldots$, and $E_{0}=\pi^{2} \hbar^{2} / 2 m^{*} L_{z}^{2}$, $k_{y}$ is the wave vector in the $y$ direction, $m^{*}$ is the effective mass of the electron, $\omega_{c}=e H / m^{*} c$ is the cyclotron frequency, $\tilde{\omega}=\sqrt{\omega_{c}^{2}+\omega^{2}}, \tilde{m}=m^{*} \widetilde{\omega}^{2} / \omega^{2}$. Moreover, $\Phi_{N}\left(x-x_{0}\right)$ is the well-known harmonic-oscillator wave function centered at $x_{0}=\widetilde{b} \widetilde{R}^{2} k_{y}$ with $\widetilde{b}=\omega_{c} / \widetilde{\omega}$ and $\widetilde{R}^{2}=\hbar / m^{*} \widetilde{\omega}$.

The FCA coefficient $\alpha$, which is related to the quantum-mechanical transition probabilities in which the carriers absorb or emit a photon with the simultaneous scattering of carriers by phonons, is given by [12]

$$
\alpha=\frac{\epsilon^{1 / 2}}{n_{0} c} \sum_{i} W_{i} f_{i}
$$

Here $\in$ is the dielectric constant of material, $n_{0}$ is the number of photons in the radiation field and $f_{i}$ is the free-carrier distribution function. The sum is over all the possible initial states ' $i$ ' of the system. The transition probabilities $W_{i}$ can be calculated using the standard secondorder Born golden rule approximation:

$$
\begin{aligned}
& W_{i}=\frac{2 \pi}{\hbar} \sum_{f q}\left[\left|\left\langle f\left|M_{+}\right| i\right\rangle\right|^{2} \delta\left(E_{f}-E_{i}-\hbar \Omega-\hbar \omega_{q}\right)+\right. \\
& \left.+\left|\left\langle f\left|M_{-}\right| i\right\rangle\right|^{2} \delta\left(E_{f}-E_{i}-\hbar \Omega+\hbar \omega_{q}\right)\right]
\end{aligned}
$$

Here $E_{i}$ and $E_{f}$ are the initial and final state energies, respectively, of electrons, $\hbar \Omega$ is the photon energy, $\hbar \omega_{q}$ is the phonon energy, and $\left\langle f\left|M_{ \pm}\right| i\right\rangle$ are the transition matrix elements from the initial state to the final state for the interaction between electrons, photons and phonons. These transition matrix elements can be represented by the following expression:

$$
\left\langle f \mid M_{ \pm} i\right\rangle=\sum_{\alpha}\left(\frac{\left\langle f\left|H_{R}\right| \alpha\right\rangle\left\langle\alpha\left|V_{s}\right| i\right\rangle}{E_{i}-E_{\alpha} \mp \hbar \omega_{q}}+\frac{\left\langle f\left|V_{s}\right| \alpha\right\rangle\left\langle\alpha H_{R} \mid i\right\rangle}{E_{i}-E_{\alpha}-\hbar \Omega}\right)
$$

where $H_{R}$ is the interaction Hamiltonian between the electrons and the radiation field, $V_{s}$ is the scattering potential due to the electron-phonon interaction.

Using the wavefunctions given by the expression (1), the matrix elements of the electron-photon interaction Hamiltonians can be written as

$$
\begin{aligned}
& \left\langle k_{y}^{\prime} N^{\prime} l^{\prime}\left|H_{R}\right| k_{y} N l\right\rangle= \\
& =-\frac{e \hbar}{m^{*}}\left(\frac{2 \pi \hbar n_{0}}{V \Omega \in}\right)^{1 / 2}(\varepsilon \kappa) \delta_{\kappa_{y} \kappa_{y}^{\prime}} \delta_{N N^{\prime}} \delta_{l l^{\prime}}
\end{aligned}
$$

where $V$ is the volume of the crystal. Here the radiation field is polarized along the wire, $\varepsilon$ is the polarization vector of the radiation field.

We shall use two different scattering processes: polar-optical scattering and acoustic -phonon scattering. The matrix elements $\left\langle k_{y}^{\prime} n^{\prime} l^{\prime}\left|V_{s}\right| k_{y} n l\right\rangle$ of electron-phonon interaction corresponding to the above two processes are equal to

$$
\left\langle k_{y}^{\prime} n^{\prime} l\left|V_{s}\right| k_{y} n l\right\rangle=C_{j}^{\prime} \delta_{k_{y}^{\prime}, k_{y} \pm q_{y}} J_{n n^{\prime}}\left(q_{x} q_{y}\right) \Lambda_{l l^{\prime}}\left(q_{z}\right)
$$

where $J_{n^{\prime}, n}\left(q_{x}, q_{y}\right)$ is the overlap integral of the harmonic wave functions:

$$
\begin{aligned}
& J_{N^{\prime}, N}\left(q_{x} q_{y}\right)= \\
& =\int_{-\infty}^{\infty} d x \exp \left(i q_{x} x\right) \Phi_{N^{\prime}}\left(x-\tilde{b} \widetilde{R}^{2} k_{y}-\tilde{b} \widetilde{R}^{2} q_{y}\right) \Phi_{N}\left(x-\tilde{b} \widetilde{R}^{2} k_{y}\right) \\
& \Lambda_{l l^{\prime}}\left(q_{z}\right)=\frac{2}{L_{z}} \int_{0}^{L_{z}} d z \exp \left(i q_{z} z\right) \sin \left(\frac{l^{\prime} \pi z}{L_{z}}\right) \sin \left(\frac{l \pi z}{L_{z}}\right) \\
& C_{j}^{\prime 2}=C_{j}^{2} F_{j}(q)
\end{aligned}
$$

The function $\Lambda_{l l^{\prime}}\left(q_{z}\right)$ given by Eq. (8) is crucial for our calculation whose suitable approximation was discussed by Ridley [28].

For the electron-polar-optic phonon interaction we have

$$
\begin{aligned}
& F_{P O L}=\frac{N_{0}^{ \pm}}{q^{2} V}, C_{P O L}^{2}=2 \pi e^{2} \hbar \omega_{0} \in^{\prime-1}, \\
& \epsilon^{\prime-1}=\left\{\frac{1}{\varepsilon_{\infty}}-\frac{1}{\varepsilon_{0}}\right\} .
\end{aligned}
$$

Here, $\varepsilon_{\infty}$ and $\varepsilon_{0}$ are the high-frequency and static dielectric constants of the semiconductor, respectively. As usual, we take phonon energy $\hbar \omega_{q}=\hbar \omega_{0} \approx$ const. 
$N_{0}=\left[\exp \left(\frac{\hbar \omega_{0}}{K_{B} T}\right)-1\right]^{-1}, N_{0}^{-}=N_{0}, N_{0}^{+}=N_{0}+1$.

where $N_{0}^{-}\left(N_{0}^{+}\right)$describes the annihilation (creation) of the phonon.

When acoustic phonon scattering is dominant, one may obtain

$C_{A C}^{2}=\frac{E_{d}^{2} K_{B} T}{2 \rho v_{s}^{2} V}, F_{A C}(q)=1$

In the case of bulk materials and at extremely strong magnetic fields, the electronic wave functions have small absolute values of momentum components parallel to the applied magnetic field. Therefore, we can neglect the $q_{z}$ dependence in the interaction potential given by $C_{j}^{\breve{y}}$.

The electron distribution function for quasi-one-dimensional nondegenrate electron gas in the presence of magnetic field can be shown as follows:

$$
\begin{aligned}
& f_{N l k_{y}}=\frac{2(2 \pi)^{1 / 2} \hbar n_{e} L_{x} L_{z} \sinh \left(\hbar \widetilde{\omega} / 2 K_{B} T\right)}{\delta\left(\widetilde{m} K_{B} T\right)^{1 / 2}} \times \\
& \times \exp \left\{-\left[\frac{(N+1 / 2) \hbar \widetilde{\omega}+l^{2} \mathrm{E}_{0}}{K_{B} T}\right]\right\} \exp \left(-\frac{\hbar^{2} k_{y}^{2}}{2 \widetilde{m}^{*} K_{B} T}\right)
\end{aligned}
$$

where $\delta=\sum_{l} \exp \left(l^{2} \mathrm{E}_{0} / K_{B} T\right), n_{e}$ is the concentration of
electrons.

Below, we shall use the following identities:

$$
\begin{aligned}
& \int_{0}^{\infty}\left|J_{n n^{\prime}}\left(q_{x}, q_{y}\right)\right|^{2} q_{\perp} d q_{\perp}=\frac{1}{R^{2}} \\
& \int_{0}^{\infty}\left|J_{n n^{\prime}}\left(q_{x}, q_{y}\right)\right|^{2} q_{\perp}^{3} d q_{\perp}=\frac{2}{R^{4}}\left(N^{\prime}+N+1\right) \\
& \int_{0}^{\infty}\left|\Lambda_{l l}\left(q_{z}\right)\right|^{2} d q_{z}=\frac{2 \pi}{d}\left(1+\frac{1}{2} \delta_{l l^{\prime}}\right)
\end{aligned}
$$

Now we make the same approximation as in [4], i.e. we take $\frac{\hbar^{2}}{2 \tilde{m}^{*}}\left(q_{y}^{2}-2 k_{y} q_{y}\right)=0$, in $\delta$ functions. Using Eqs (4-6) and (9) in (3) and also identities (11), we obtain the following expression for the FCA coefficient for polar and acoustic phonon scattering in a Q1D semiconducting structure in the presence of a magnetic field:

$$
\begin{aligned}
& \alpha_{P O L}(H)=\frac{4 \pi^{2} e^{4} \hbar \omega_{0} n_{e} \sinh \left(\hbar \widetilde{\omega} / 2 K_{B} T\right)}{c \epsilon^{1 / 2} \in^{\prime} m^{* 2} \Omega^{3} L_{z} \widetilde{b} R^{2} \delta} \times \\
& \times \sum_{N_{f} l_{f}} \sum_{N_{i} l_{i}}\left(1+\frac{\delta_{l_{f} l_{i}}}{2}\right) \exp \left\{-\frac{1}{K_{B} T}\left[\left(N_{i}+\frac{1}{2}\right) \hbar \widetilde{\omega}+l_{i}^{2} E_{0}\right]\right\} \times \\
& \times\left\{N_{0} \delta\left(\left(N_{f}-N_{i}\right) \hbar \widetilde{\omega}+\left(l_{f}^{2}-l_{i}^{2}\right) E_{0}-\hbar \Omega+\hbar \omega_{0}\right)+\right. \\
& \left.+\left(N_{0}+1\right) \delta\left(\left(N_{f}-N_{i}\right) \hbar \widetilde{\omega}+\left(l_{f}^{2}-l_{i}^{2}\right) E_{0}-\hbar \Omega-\hbar \omega_{0}\right)\right\}
\end{aligned}
$$

$\alpha_{A C}(H)=\frac{2 \pi e^{2} E_{d}^{2} n_{e}\left(K_{B} T\right)^{1 / 2} \sinh \left(\hbar \widetilde{\omega} / 2 K_{B} T\right)}{c \rho \epsilon^{1 / 2} v_{s}^{2} m^{* 2} \Omega^{3} L_{z} \widetilde{b}^{2} R^{4} \delta}$

$\sum_{N_{f} l_{f}} \sum_{N_{i} l_{i}}\left(1+\frac{\delta_{l_{f} l_{i}}}{2}\right)\left(N_{f}+N_{i}+1\right) \times$

$\times \exp \left\{-\frac{1}{K_{B} T}\left[\left(N_{i}+\frac{1}{2}\right) \hbar \widetilde{\omega}+l_{i}^{2} E_{0}\right]\right\} \times$

$\times\left\{\delta\left(\left(N_{f}-N_{i}\right) \hbar \widetilde{\omega}+\left(l_{f}^{2}-l_{i}^{2}\right) E_{0}-\hbar \Omega\right)\right\}$.

It is particularly convenient to express our results in terms of the dimensionless ratio of the FCA coefficient in presence of the magnetic field to that in the absence of the field. For scattering through acoustic phonon, we adopt the results [24]

$\alpha_{A C}(0)=\frac{2^{3 / 2} e^{2} E_{d}^{2}\left(K_{B} T\right)^{3 / 2} n_{e} \sinh \left(\hbar \omega / 2 K_{B} T\right)}{m^{* 1 / 2} l_{\omega}(\hbar \Omega)^{3} \in^{1 / 2} \rho v_{s}^{2} c L_{z} \delta} \times$

$\times \sum_{n_{f} l_{f}} \sum_{n_{i} l_{i}}\left(1+\frac{1}{2} \delta_{l_{i} l_{f}}\right) \exp \left(-\frac{\left(n_{i}+1 / 2\right) \hbar \omega+l^{2} E_{0}}{K_{B} T}\right) \times$

$\times Z \exp (Z) K_{1}(Z)$

where

$Z=\frac{\hbar \Omega-\left(n_{f}-n_{i}\right) \hbar \omega-\left(l_{f}^{2}-l_{i}^{2}\right) \mathrm{E}_{0}}{2 K_{B} T}$

and $K_{1}(x)$ is the modified Bessel function of the second kind, $l_{\omega}^{2}=\hbar / m^{*} \omega$. In the quantum limit, in which only the $n_{i}=n_{f}=l_{i}=l_{f}=1$ quantum level is occupied and $\hbar \omega_{c}>>K_{B} T$, only the lowest Landau level $N=0$ is thermally populated, the ratio $\alpha_{A C}(H) / \alpha_{A C}(0)$ takes the particularly simple form

$\frac{\alpha_{A C}(H)}{\alpha_{A C}(0)}=$

$=\frac{2 \hbar^{1 / 2} \widetilde{\omega}^{4}\left(K_{B} T\right)^{1 / 2} \exp \left(-\hbar \widetilde{\omega} / 2 K_{B} T\right)}{\Omega \omega^{1 / 2} \omega_{c}^{2} K_{1}\left(\hbar \Omega / 2 K_{B} T\right)} \times$

$\times \sum_{N_{f}}\left(N_{f}+1\right) \delta\left(N_{f} \hbar \widetilde{\omega}-\hbar \Omega\right)$

For optical phonon scattering, the ratio takes a similar form

$\frac{\alpha_{P O L}(H)}{\alpha_{P O L}(0)}=F\left(T, \omega_{c}, \Omega\right)$

From Eqs (15)-(16), it can be seen that, the ratio depends only upon the magnetic field, absolute temperature, and photon frequency and does not depend upon such material parameters as the values of the deformation potential, sound velocity, or density of the material, although, of course, the absolute value of absorption coefficient does depend upon the numerical values of these parameters. 


\section{G.B. Ibragimov: Theory of free-carrier absorption in the presence of ...}

\section{Discussion}

Thus, we have obtained general expressions for FCA coefficients for QWWs in the presence of the quantizing magnetic field. From Eqs (12)-(13) it can be seen that, in the extreme quantum limit $\left(\hbar \widetilde{\omega}>>K_{B} T, N_{i}=0, l_{i}=l_{f}=1\right)$ for polar optical phonons, the FCA coefficient oscillates as a function of the magnetic field and photon frequency with resonances occuring when $P \widetilde{\omega}=\Omega \pm \omega_{0}$. Since $\omega_{c}<\widetilde{\omega}$, for $\omega>0$, the resonances are shifted to smaller magnetic fields. For $\omega=0$, i.e., in the absence of confinement, $\widetilde{b}^{2}=1, \widetilde{\omega}=\omega_{c}$, and we recover the usual resonance condition $P \omega_{c}=\Omega \pm \omega_{0}$. For the elastic scattering by acoustic phonons, resonances are expected when $P \widetilde{\omega}=\Omega_{0}$.

The oscillatory dependence of the absorption on magnetic field can be understood in terms of the Landau subband structure of the electronic energy levels in quantizing magnetic fields. As the magnetic field, and therefore $\widetilde{\omega}$, increases there are fewer and fewer subbands to which the transition can place until finally. Every time that the ratio $\left(\Omega \pm \omega_{0}\right) / \widetilde{\omega}$ equals an integer value, the transition can take place with an additional subband ending as a final state.

In conclusion, we predict that FCA coefficient should increase with magnetic field with an oscillatory dependence on the field when $\Omega>\widetilde{\omega}$. The magnetic field dependence of the FCA coefficient is explained in terms of the field dependence of the scattering rates and the possibility of phonon-assisted transitions between various Landau levels when $\Omega>\widetilde{\omega}$.

\section{Acknowledgment}

The author would like to thank Prof. M.I. Aliev and Prof. F.M. Gashimzade for helpful discussions.

\section{References}

1. J.A. Brum and G. Bastard, Superlattices Microstruct. 4, p. 443 (1998).

2. F.M. Peeters, Quantum Hall Resistance in the Quasi-OneDimensional Electron Gas // Phys. Rev. Lett., 61, p. 589 (1989).

3. H. Akera and T. Ando, Quantum Hall effect in quantum wires // Phys. Rev. B 39, p. 5508-5511 (1989).

4. P. Vasilopoulos, P. Warmenbol, F.M. Peeters and J.T. Devreese, Magnetophonon resonances in quasi-one-dimensional wires // Phys. Rev. B, 40, p. 1810-1816 (1989).

5. U. Bockelmann and G. Bastard, Interband absorption in quantum wires. II. Nonzero-magnetic-field case // Phys. Rev. B, 45, p. 1700-1704 (1992)

6. C.W.J. Beenakker and H. van Houten // Solid State Phys., 44, p. 1 (1991).

7. W. Wegscheider et.al. // Phys. Rev. Lett. 71, p. 4071 (1990).

8. F. Vouilloz, D.Y. Oberli, M-A. Dupertuisi, A. Gustofsson, F. Recnhardt and E. Kapon. Effect lateral confinement on valence-band mixing and polarization anisotropy in quantum wires // Phys. Rev. B, 57, p. 12378 (1998).
9. R. Cingolani, F. Sogana, Y. Arakawa, R. Rinaldi, M.De. Vittorio, A. Posscase, A. Towrino, M. Gatalino, L. Vasanelli. Microphotoluminescence spectroscopy of vertically stacked in $\mathrm{In}_{1-\mathrm{x}} \mathrm{Ga}_{\mathrm{x}}$ As quantum wires // Phys. Rev., 58, p. 1962-1966 (1998).

10. M. Lomascolo, P. Ciccarese, R. Cingolani and R. Rinaldi Free versus localized exciton in GaAs V-shaped quantum wires // J. Appl. Phys., 83, p. 302-305 (1998).

11. K. Seeger, Semiconductor Physics: An Introduction (Springer, New York, 1997), 6th ed.

12. H.N. Spector, Free-carrier absorption in quasi-two-dimensional semiconducting structures // Phys. Rev. B, 28, p. 971976 (1983).

13. H. Adamska and. H.N. Spector, Free-carrier absorption in quantum well structures for polar optical phonon scattering // J. Appl. Phys., 56, p. 1123-1127 (1984).

14. C. Trallero Ciner and M. Anton, Quantum Theory of FreeCarrier Absorption in Quasi-Two-Dimensional Semiconducting Structures // Phys. Stat. Sol (b), 133, p. 563-572 (1986).

15. V.L. Gurevich, D.A. Parshin and K.E. Stengel // Fiz.Tverd. Tela, 30, p. 1468 (1988).

16. J.S. Bhat, S.S. Kubakaddi and B.G. Mulimani, Free carrier absorption in semiconducting quantum wells for confined LO phonon scattering // J. Appl. Phys., 72, p. 4966-4968 (1992).

17. C.C. Wu and C.J. Lin Free-carrier absorption in n-type gallium arsenide films for polar optical phonon scattering // $J$. Appl. Phys., 79, p. 781-785 (1996).

18. C.C. Wu and C.J. Lin, Free-carrier absorption in $n$-type piezoelectric semiconductor films. // J. Phys: Condens matter, 6, p. 10147-10156 (1994).

19. F.M. Gashimzade and E.V. Tahirov, Free-Carrier Absorption in Quantum-Well Structures for Charged Impurity Scattering // Phys. Stat. Sol (b), 160, K177-K181 (1990).

20. I. Vurgaftman and J.R. Meyer TE-and TM-polarized roughness-assisted free-carrier absorption in quantum wells at midinfrared and terahertz wavelengths // Phys. Rev. B, 60, p. 14294-14301 (1999).

21. G.G. Zegrya and V.E. Perlin, Intraband light absorption in quantum wells at the expense of electron-electron collisions // Fiz. Tekh. Poluprovodn., 32, p. 466-471 (1998).

22. G.B. Ibragimov, Free-carrier absorption in semiconducting quantum wells for alloy-disorder scattering // J. Phys.: Condens. Matter, 14, p. 4977-4983 (2002).

23. G.B. Ibragimov, Free-carrier absorption in size-confined systems in a quantizing magnetic field // Fizika, 6, p. 43-46 (2000).

G.B. Ibragimov, Free-carrier magnetoabsorption in quantum well structures // Ukr. J. Phys., 48, p. 527-532 (2003).

24. S.S Kubakaddi and B.G Mulimani, Free-carrier absorption in semiconducting quantum well wires // J. Phys. C: solid State Phys. 18, p. 6647-6652 (1985).

25. H. Adamska and N. Spector, Free-carrier absorption from electrons in confined systems // J. Appl. Phys., 59, p. 619-626 (1986).

26. G.B. Ibragimov, Free-carrier absorption in semiconducting quantum well wire for alloy-disorder scattering // J. Phys.: Condens. Matter, 14, p. 8145-8152 (2002)

27. G.B. Ibragimov, Theory free-carrier absorption in quantum wires at the expense boundary roughness scattering // Semiconductor Physics, Quantum Electronics\& Optoelectronics, 6, (1), p. 9 (2003).

28. B.K. Ridley, The electron-phonon interaction in quasi-twodimensional semiconductor quantum-well structures // $J$. Phys. C: Solid State Phys. 15, p. 5899-5917 (1982). 